\title{
A New Scheme for UAV TT\&C and Information Transmission System
}

\author{
Yongchuan WANG, Xijun GAO, Yongke LI, Qingmin TIAN \\ Department of Unmanned Aerial Vehicle Engineering, Ordnance Engineering College, \\ Shijiazhuang, 050003, China \\ email: mrwyc@sina.com
}

Keywords: UAV; TT\&C and information transmission; OFDM; SC-FDE; MIMO

\begin{abstract}
The paper assume a new scheme for UAV TT\&C and information transmission system, according to the function and performance requirement of UAV TT\&C and information transmission system and the characteristic of ground-air wireless channel of UAV system, and combing with the key technologies of wireless communications, OFDM, SC-FDE, MIMO, Cooperative Communication and Cognitive Radio. The theory model frame and the qualitative performance analysis of the scheme are given.
\end{abstract}

\section{Introduction}

UAV integrated TT\&C system was made in China on 1980's. The system function includes telecommand, telemetry, tracking and data transmission. It was a very advanced TT\&C and information transmission system scheme then. From then on, a new UAV TT\&C and information transmission system was set up every three to five years. Although the new system had ameliorated, the improvement of institution was still less. The requirements of dependability, effectiveness and security are higher with the appearance of new UAV and the complication of electromagnetism environmental of UAV war field.

Recently, the wireless communication was growing by leaps and bounds, and the technologies of OFDM, SC-FDE, MIMO, cooperation communication and cognition radio were becoming more mature. They were considered as the key technologies of next generation wireless communication system or network generally, and also were applied in wireless communication or standard of civilian or military uses. The new way was provided by the present and development of the technologies for constructing new UAV TT\&C and information transmission system.

In the paper, according to the function and performance requirement of UAV TT\&C and information transmission system and the characteristic of ground-air wireless channel of UAV system, and combing with the key technologies, a new UAV TT\&C and information transmission scheme was assumed. The theory model frame of the scheme and the qualitative analysis of the performance were given.

\section{UAV TT\&C and information transmission system}

UAV TT\&C and information transmission system which was composed of data-link and ground-control- station was key part of UAV system. It was used to achieve telecommand, telemetry, tracking location and load information transmission, and also could control the UAV remotely or get the real-time reconnaissance information. The performance and scale of UAV system were decided by the UAV TT\&C and information transmission systems' to a great extent. The UAV TT\&C and information transmission system which could afford high-speed data transmission dependability and also had the performance of strong survivability, anti-jamming and anti-intercept was differ from the common wireless communication.

\section{The channel characteristic of UAV-to-ground}

The wireless channel of UAV-to-ground was the very complicated and dicky time-varying channel[1,2,3]. The Doppler effect, multi-path effect and angle dispersion were also existed in the 
channel which contain the transmission loss, rain fade and shadow effect. The transmission loss, rain fade and shadow effect could come into being large-scale fading. And the large-scale fading is the considerable factor for line budget, outage probability analysis and channel model foundation of the system designing. The Doppler effect, multi-path effect and angle dispersion could come into being small-scale fading. The small-scale fading could bring the distortion to the receiving signal in the time domain, frequency domain and airspace which affected the dependability of signal transmission. So the small-scale fading should be considered when the physical layer of data-link was confirmed.

\section{The new scheme model of UAV TT\&C and information transmission}

\subsection{The component and application of UAV system}

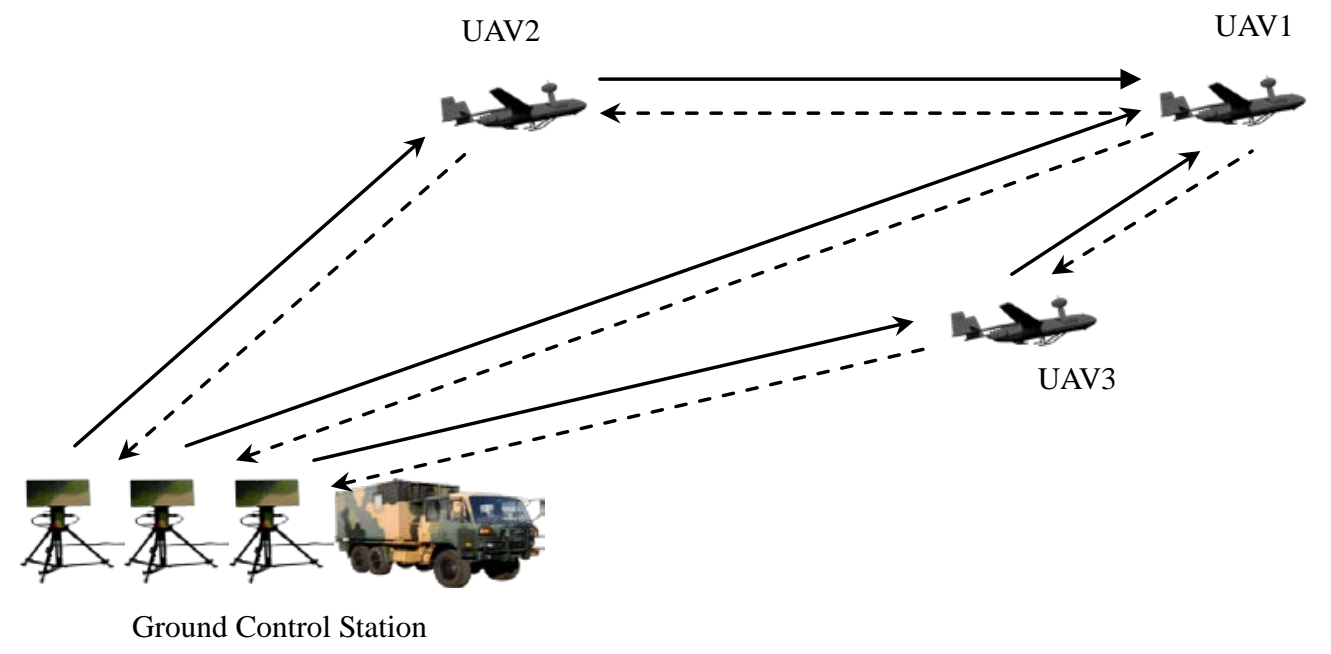

Fig.1 Structure sketch of UAVs

The component and application of UAV system was assumed as Fig.1. The ground station set multi-antennas which can transmit data with multi-UAVs simultaneity. The every UAV sets one antenna only which can transmit data with ground control station and also other UAVs. For example the data transmission of down telemetry, when the multi-UAVs are working at the same time, the one of the UAV can transmit data to ground control station directly and also can transmit data through the second and the third UAVs as relaying to ground control station which forms the co-operation relaying network of down telemetry. The UAV configure cognition radio system for cognizing wireless electromagnetism environment. The cognition radio system can cognize and forecast the channel environment and condition between the UAV and the ground control station or among UAVs for selecting the channel and relaying adaptively. Then the UAV can transmit telemetry data to the ground control station whenever and wherever possible. The up-telecommand data transmission has the similar way as the downlink.

\subsection{The uplink and downlink configuration model}

The up-telecommand link of UAV data-link adopts OFDM transmission and the down-telemetry link uses SC-FDE transmission. The transceiver configuration model of the ground control station is shown as Fig.2. And the transceiver configuration model of UAV is shown as Fig.3. In the figures, the broken line is effective when the up-telecommand or down-telemetry transmit data through co-operation relaying. 


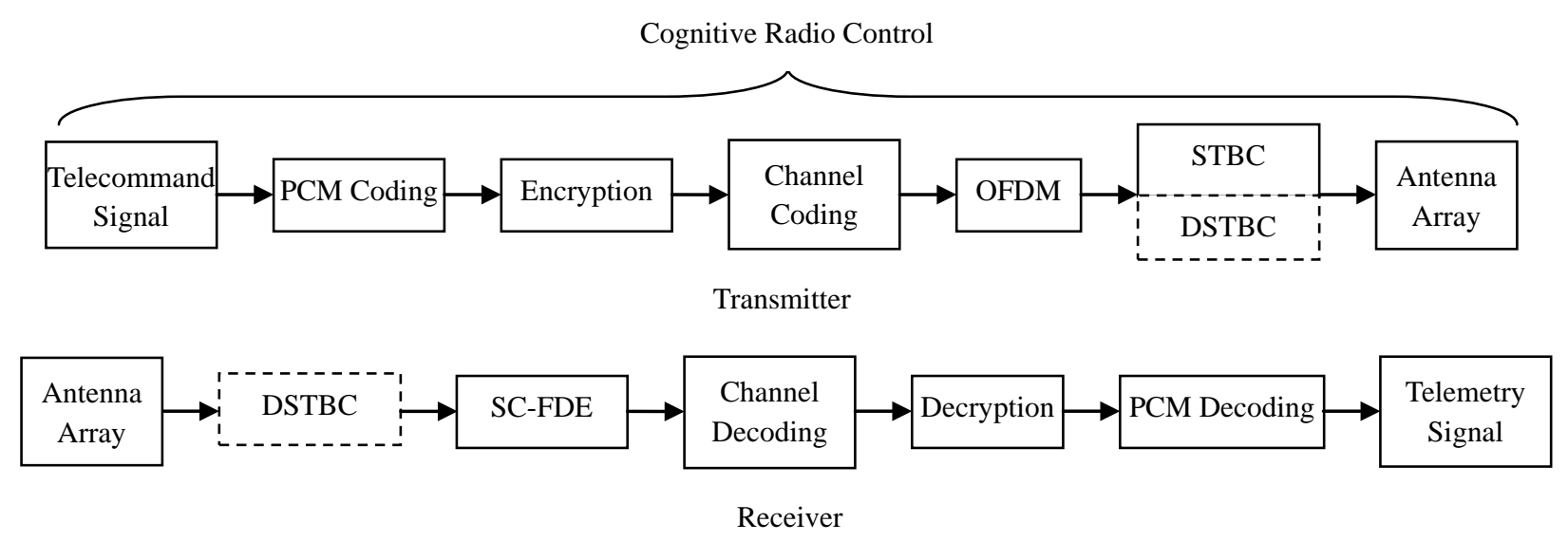

Fig.2 Transreceiver principle model of ground control station

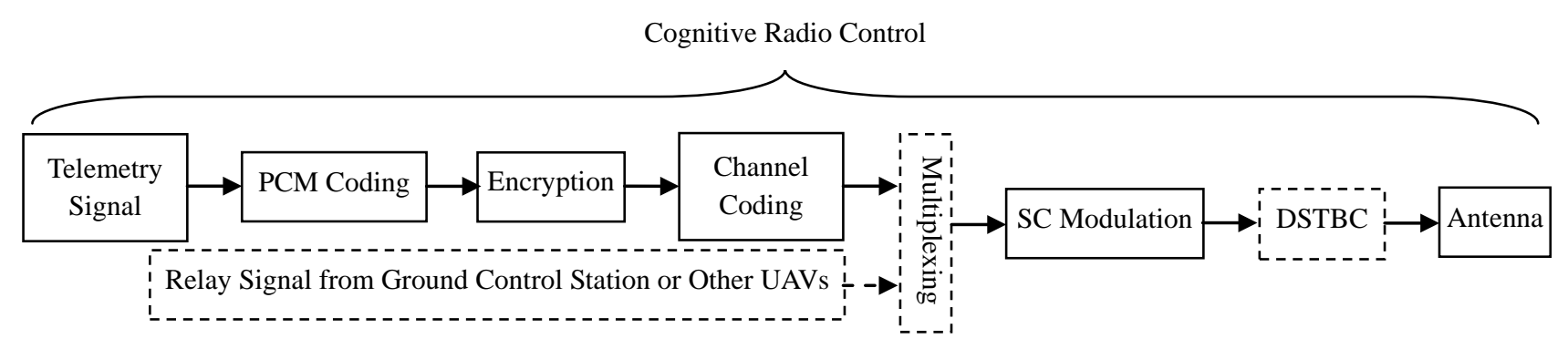

Transmitter

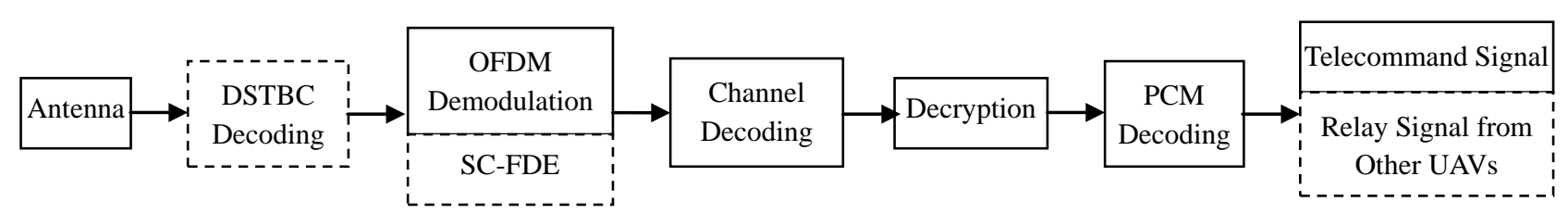

Receiver

Fig.3 Transreceiver principle model of UAV

\subsection{The analysis of the new UAV TT\&C and information transmission scheme}

The OFDM is a good modulation technology for the high-speed up-telecommand link of the high-speed UAV. It can overcome the multipath effect and achieve frequency diversity. The multipath reflection of $30 \mathrm{~km}$ and time delay spread under $100 \mu \mathrm{s}$ can be tolerated. And also the user capacity is larger than the conventional frequency system of single carrier wave spread which the user number is twice to tenth the conventional frequency system of single carrier[4]. The research fruit which was released by the laboratory of Canada Pacific Ocean microwave Research Center in the year of 2003 is that the OFDM system in the tactics UAV data-link could confront with the multi-path interference and communicate through non-LOS when the sub-carrier was QPSK or 16QAM, the signal bandwidth was $8 \mathrm{MHz}$, the transmission rate was $5 \mathrm{Mbps}$ to $20 \mathrm{Mbps}$ according to the different coding efficiency and modulatiion way of sub-carrier[5].

When the UAV is far from the ground control station, the low elevation of ground-to-air and the obvious multi-path effect are the adverse factors for the common communication technology. But the multi-path can be considered as advantageous factor for the MIMO. The MIMO regards the multi-path wireless channel, transmitter and receiver as an integer to joint optimization. It can improve the information transmission rate and the utilization of frequency spectrum. And it can improve the reliability of signal transmission through increasing information redundancy. And also it can seek for the reasonable compromise between them[6]. In the year of 2006, MIMO was applied in aero-telemetry by Chris Potter et al. of telemetry science center of the Missouri Science and Technology University USA. And they developed a set MIMO system of $2 \times 2$ [5]. 
The space of UAV is small. So the multi-antennas are not easy to be fixed. Then, the cooperation diversity technology can be adopted to unite many UAVs with only one antenna for forming cooperation relaying network. And it also can be considered as virtual MIMO system which can obtain capacity gain and reliability gain by the STBC of 3G system which is simpleness and has well performance[7]. In the year of 2011, Charles of the laboratory of USA Air Force reported the research fruit which the technology of OFDM with STBC was applied in the cooperation communication network of microminiature UAV, then the data rate could be up to $1 \mathrm{MHz}$ in the frequency band of 300 to $500 \mathrm{MHz}$ when the sub-carrier was DBPSK or DQPSK[8].

The SC-FDE system has the similar frequency band efficiency, anti-fading performance and computation complexity to the OFDM. But the SC-FDE system that transfers a majority of signal processing and cost to receiver is different from OFDM system. So it is fit for the down telemetry link greatly and mitigates the burden of UAV airborne platform[9]. Basing on this advantage, the SC-FDE was adopted as physical layer technology from MS mobile station to base station in the standard of 3GPP LTE and 4G. The cooperation relaying strategy of decoding transmission is adopted in the model of UAV receiver of Fig.3. If the cooperation relaying strategy of amplification transmission is adopted, the receiver can be simpler.

If the cognition radio technology is adopted in UAV data-link system, the data-link can cognize electromagnetism frequency spectrum of ambience and forecast channel transmission condition. When the system is confront for the jamming, the transmission parameters can be changed automatically to avoid jamming. When the worst transmission channel condition is forecasted, the channel can be discarded to choose better channel for relaying transmission or cooperation transmission[11]. The data transmission network of UAV cooperation relaying with cognition radio can increase the difficulty of enemy detection, intercept and capture, detection observer and control and information transmission and advance the survivability, anti-jamming and the performance of anti-intercept[10,11].

\section{Conclusion}

OFDM, SC-FDE, MIMO, cooperation communications and cognition radio are the key and new technologies. In the paper, these technologies were discussed to apply in UAV TT\&C and information transmission system, and a integrated UAV TT\&C and information transmission institution was assumed. The theory model frame of the institution and the qualitative analysis of the performance were given. The new institution can improve the system capacity and reliability and assure the performance of strong survivability, anti-jamming and anti-intercept. The research of new institution was only based on theory lay in the paper. So the further research on system simulation and implement should be last.

\section{References}

[1] GAO Bao-sheng,ZHU Liang-bin. Channel characteristics of UAV ground-to-air datalink and broadband data transmission[J]. Radio Engineering of China, 2011, 41(9): 4-6

[2] Guo Yi, Ge Jianhua, Liu Gang. Simulation implementation of the aeronautical channel in dynamic environment with low elevation angle [C]. 2010 International Conference on Computer Application and System Modeling, Taiwan, October 22-24, 2010: V12-379- V12-383

[3] Erik Haas. Aeronautical channel modeling [J]. IEEE Transaction on Vehicular Technology, 2002, 51(2): 254-264

[4] LUO Ren-ze. Key techniques of new generation wireless mobile communication systems [M]. Beijing: Beijing University of Posts and Telecommunications Press, 2007: 1-2

[5] ZHAO Hai-long, ZHANG Jian, ZHOU Jie. Key techniques of next generation wireless communication and their applications in telemetry [J]. Information and Electronic Engineering, 
2012, 10(2): 1-6

[6] LIU Ju, XU Hong-ji, ZHENG Li-na. Space-time and cooperative signal processing in wireless communications [M]. Beijing: Posts and Telecom Press, 2014: 2-2

[7] Naguib A F, Seshari N, and Calderbank A R. Increasing data rata over wireless channels [J]. IEEE Signal Processing Magazine, 2000, 17(3):76-92

[8] Charles W. Bostian, Alexander R. Young. The application of cognitive radio to coordinated unmanned aerial vehicle missions [EB/OL]. USA air force research laboratory technical report, 2011, http://www.dtic.mil/dtic/tr/fulltext/ui/a546145.pdf

[9] F.Pancaldi, G.Vitetta, and R.Kalbasi. Single-carrier frequency domain equalization [J]. IEEE Signal Processing Magazine, 2008, 25(5): 37-56.

[10] Xiaohua Li and Wednel Cadeau. Anti-jamming performance of cognitive radio networks [C]. 2012 46th Annual Conference on Information Sciences and Systems, Princeton, NJ, March 21-23, 2012:1-6

[11] R. Chen, l-M. Park, and J. H. Reed. Defense against primary user emulation attacks in cognitive radio networks [J]. IEEE Journal on Selected Areas in Communication, Special Issue on Cognitive Radio Theory and Applications, 2008, 26(1):25-37 